\title{
Outstanding X-ray emission from the stellar radio pulsar CU Virginis
}

\author{
J. Robrade ${ }^{1}$, L. M. Oskinova ${ }^{2,3}$, J. H. M. M. Schmitt ${ }^{1}$, P. Leto $^{4}$, and C. Trigilio ${ }^{4}$ \\ ${ }^{1}$ Hamburger Sternwarte, University of Hamburg, Gojenbergsweg 112, 21029 Hamburg, Germany \\ e-mail: jrobrade@hs.uni-hamburg.de \\ 2 Institute for Physics and Astronomy, University Potsdam, 14476 Potsdam, Germany \\ 3 Kazan Federal University, Kremlevskaya Str 18, Kazan, Russia \\ ${ }^{4}$ INAF - Osservatorio Astrofisico di Catania, Via S. Sofia 78, 95123 Catania, Italy
}

Received 24 May 2018 / Accepted 6 August 2018

\begin{abstract}
Context. Among the intermediate-mass magnetic chemically peculiar (MCP) stars, CU Vir is one of the most intriguing objects. Its $100 \%$ circularly polarized beams of radio emission sweep the Earth as the star rotates, thereby making this strongly magnetic star the prototype of a class of nondegenerate stellar radio pulsars. While CU Vir is well studied in radio, its high-energy properties are not known. Yet, X-ray emission is expected from stellar magnetospheres and confined stellar winds.

Aims. Using X-ray data we aim to test CU Vir for intrinsic X-ray emission and investigate mechanisms responsible for its generation. Methods. We present X-ray observations performed with XMM-Newton and Chandra and study obtained X-ray images, light curves, and spectra. Basic X-ray properties are derived from spectral modelling and are compared with model predictions. In this context we investigate potential thermal and nonthermal $\mathrm{X}$-ray emission scenarios.

Results. We detect an X-ray source at the position of CU Vir. With $L_{\mathrm{X}} \approx 3 \times 10^{28} \mathrm{erg} \mathrm{s}^{-1}$ it is moderately X-ray bright, but the spectrum is extremely hard compared to other Ap stars. Spectral modelling requires multi-component models with predominant hot plasma at temperatures of about $T_{\mathrm{X}}=25 \mathrm{MK}$ or, alternatively, a nonthermal spectral component. Both types of model provide a virtually equivalent description of the X-ray spectra. The Chandra observation was performed six years later than those by XMM-Newton, yet the source has similar X-ray flux and spectrum, suggesting a steady and persistent X-ray emission. This is further confirmed by the $\mathrm{X}$-ray light curves that show only mild X-ray variability.

Conclusions. CU Vir is also an exceptional star at X-ray energies. To explain its full X-ray properties, a generating mechanism beyond standard explanations, like the presence of a low-mass companion or magnetically confined wind-shocks, is required. Magnetospheric activity might be present or, as proposed for fast-rotating strongly magnetic Bp stars, the X-ray emission of CU Vir is predominantly auroral in nature.
\end{abstract}

Key words. stars: individual: CU Vir - stars: activity - stars: chemically peculiar - stars: magnetic field - X-rays: stars

\section{Introduction}

The A0p star CU Vir (HD 124224, HR 5313) is an enigmatic star of the upper part of the main sequence located at a distance of about $79 \mathrm{pc}$ (van Leeuwen 2007). With its $V=5.0 \mathrm{mag}$, it is among the most prominent nearby intermediate mass magnetic chemically peculiar (MCP) stars, also termed Ap/Bp stars (p: peculiar), and shows pronounced photometric and spectroscopic variability (see e.g., Babcock 1958). Furthermore, it is so far a unique main sequence star that shows regular radio pulses persisting over decades, resembling the radio lighthouse of pulsars and interpreted as auroral radio emission similar to those observed on planets (see e.g., Trigilio et al. 2000, 2011). In contrast to the rotationally modulated gyrosynchrotron radio emission commonly observed in MCP stars, the $100 \%$ right-handed circularly polarized radio pulses from CU Vir are explained by the electron cyclotron maser emission (ECME) mechanism. The polarization sense indicates that these originate from the northern polar regions of the oblique stellar magnetosphere, where annular rings emit narrow radio beams that sweep over the Earth location twice per stellar rotation. With a period of about $P_{\text {rot }}=0.52 \mathrm{~d}, \mathrm{CU}$ Vir is an unusually fast rotator for its class, and shows, in addition, alternating variability of its rotation period over decades (Mikulášek et al. 2011; Krtička et al. 2017).

Beside numerous radio studies, several dedicated multiwavelength campaigns on CU Vir have been executed. A detailed study of its variability in optical/UV emission and its spectral energy distribution (SED) showed that this variability can be explained by strong spots of elemental over- and under-abundances (Krtička et al. 2012). A mapping of CU Vir's abundance anomalies and magnetic field based on spectropolarimetric observations as well as a reassessment of the stellar parameters was recently performed by Kochukhov et al. (2014). According to this study, CU Vir is viewed under an inclination of about $i=46^{\circ}$, and its magnetic dipole axis is tilted by about $\beta=79^{\circ}$ to the rotational axis and has a field strength of $B_{\mathrm{d}}=3.8 \mathrm{kG}$. The derived magnetic maps show a dipolarlike field topology that is nonaxisymmetric with large differences between regions of opposite polarity, providing a natural explanation for the north-south asymmetry in the observed radio pulses.

The spectral classification of CU Vir (B9p/A0p) as well as its stellar parameters vary slightly in the literature; recent values as determined by Kochukhov et al. (2014) are $M=3.1 M_{\odot}$, 
$R=2.1 R_{\odot}$ and $T_{\text {eff }}=12750 \mathrm{~K}$. Using $v \sin i=145 \mathrm{~km} \mathrm{~s}^{-1}$ with $i=46^{\circ}$ implies a rotational speed of about $200 \mathrm{~km} \mathrm{~s}^{-1}$. While rotating rapidly, probably due to its youth, CU Vir is still nearly spherical and effects of gravitation darkening are moderate with temperature contrast of only a few $100 \mathrm{~K}$.

The existence of intrinsic X-ray emission from late-B and early-A stars has been debated, as "normal" late-B/early-A stars are expected to be virtually X-ray dark, since these stars neither drive magnetic activity nor strong stellar winds. Often, lowmass coronal companions are suspected and identified as the true $\mathrm{X}$-ray source. However, intrinsic X-ray emission from magnetically confined wind shocks (MCWS) could also be expected in the $\mathrm{Ap} / \mathrm{Bp}$ stars. In this model, originally proposed to explain the X-ray emission of the A0p star IQ Aur (Babel \& Montmerle 1997), the stellar wind from both hemispheres is channelled by the magnetic field and collides in the vicinity of the equatorial plane, leading to strong shocks and thereby plasma heating to $\mathrm{X}$-ray temperatures. Advanced MCWS models, using magnetohydrodynamic simulations (MHD), or rigid rotating magnetosphere models (RRM) are now "standard" models and have been used to interpret the observed X-ray properties of magnetic massive stars (e.g., ud-Doula et al. 2014).

A three-dimensional (3D) model able to simulate the incoherent gyrosynchrotron radio emission from a typical rapidly rotating magnetosphere of a hot magnetic star has previously been developed (Trigilio et al. 2004). Using this model, the multiwavelength radio light curves of CU Vir have been simulated, constraining the magnetospheric physical conditions (Leto et al. 2006). Recently, the same simulation approach has also been successfully applied to the cases of the fast-rotating and strongly magnetic B2Vp stars HR 7355 and HR 5907 (Leto et al. 2017, 2018). These two stars show evidence of nonthermal X-ray emission. By using the 3D model, computed to simulate the gyrosynchrotron radio emission of these two stars, it was shown that in addition to thermal plasma heated by the shocked magnetically confined wind streams, a nonthermal auroral X-ray radiation is also expected. Assuming a common framework able to explain the radio emission features of the hot magnetic stars, it is expected that similar auroral X-ray emission shall be observable also from CU Vir.

The star CU Vir was observed by XMM-Newton in 2011 and detected in X-rays (Robrade 2016), but the combination of positional offset and blurred point spread function (PSF) made confirmation of the detection desirable. For this purpose a Chandra ACIS observation was initiated by us and performed in 2017. Here we present a detailed analysis of the available X-ray data from CU Vir and put it into the context of current models to explain the X-ray emission in magnetic intermediate-mass stars.

\section{Observations and data analysis}

The target CU Vir was observed with XMM-Newton for about $30 \mathrm{ks}$ in 2011 (ToO, PI: Schartel) and we use data from the EPIC (European Photon Imaging Camera), consisting of the pn and two MOS detectors (the acronyms refer to the CCD type). All detectors were operated with the thick filter to avoid optical contamination. The Optical Monitor (OM) was blocked and the Reflection Grating Spectrometer (RGS) produced no useful data due to the low source flux. Motivated by X-ray excess photons present in the XMM-Newton data, we initiated a re-observation with Chandra (PI: Robrade) that was performed in 2017 with the ACIS-S detector (Advanced CCD Imaging Spectrometer) in the $1 / 4$ array configuration. While XMM-Newton has a higher sensitivity, Chandra has a much better spatial resolution and is less
Table 1. X-ray observation log for CU Vir.

\begin{tabular}{lrrr}
\hline \hline Mission & Obs. ID. & Start-date & Texp (ks) \\
\hline XMM-Newton & 0677980501 & $2011-07-17$ & 27 \\
Chandra & 18925 & $2017-05-13$ & 29 \\
\hline
\end{tabular}

prone to background events. The two observations complement each other very well.

A detailed description of the instruments can be found on the respective mission websites ${ }^{1}$; the observations used are summarized in Table 1.

The XMM-Newton data were reprocessed with the XMMNewton Science Analysis System software SAS 16.1 (de la Calle 2018) and standard SAS tools were used to produce images, light curves, and spectra. The Chandra data processing used the CIAO 4.9 software package (Fruscione et al. 2006) and again standard tools were used to produce images, light curves, and spectra. Source photons were extracted from circular regions and background was taken from nearby regions. The XMM-Newton detectors cover the energy range $0.2-10.0 \mathrm{keV}$, while ACIS-S covers nominally the $0.3-10.0 \mathrm{keV}$ range, but its effective area below $0.5 \mathrm{keV}$ is very small.

Spectral analysis was carried out with XSPEC V12.9 (Arnaud et al. 1996) and we used multi-temperature APEC (Smith et al. 2001) with solar abundances as well as APEC + powerlaw models to fit the X-ray spectra. The used spectral models assume a solar abundance pattern and ignore a potential absorption component as it was found to be consistent with zero. Adding further spectral components or introducing additional free parameters does not improve the models significantly and results in poorly constrained values. For the XMM-Newton observation, the pn and the combined MOS spectrum are modeled simultaneously. All spectra were rebinned to a minimum of five counts per bin and model optimization used the "cstat" algorithm, applicable for Poisson-distributed data. This approach allows us to treat the data from the different detectors in an identical fashion without loosing a significant amount of spectral resolution. We tested different binning and modeling approaches and obtained very similar results, indicating the robustness of our general findings. Given errors denote the $90 \%$ confidence range.

\section{Results}

Here we report on the results obtained from the X-ray observations of CU Vir, subdivided into separate topics.

\subsection{X-ray images and light curves}

A clear photon excess at the expected source position is present in both X-ray images of CU Vir shown in Fig. 1. The XMMNewton source photon distribution is blurred and its photon centroid is about $1.5^{\prime \prime}$ offset from the optical position. While this is not uncommon for XMM-Newton pn (EEF80/1 keV is about $20^{\prime \prime}$ ), with EEF80 denoting an encircled energy fraction of $80 \%$, for a clear association of the detected X-ray photons to a single source at the position of CU Vir, a confirmation is desirable. Chandra is well suited for this exercise (EEF80/1 keV is about $0.5^{\prime \prime}$ ) and the ACIS-S image shows a source that is pointlike at the spatial resolution of the detector. Its position matches the optical position at about $0.4^{\prime \prime}$, which is a typical value for

1 https://www. cosmos. esa. int/web/xmm-newton; http://cxc. harvard.edu/index.html 

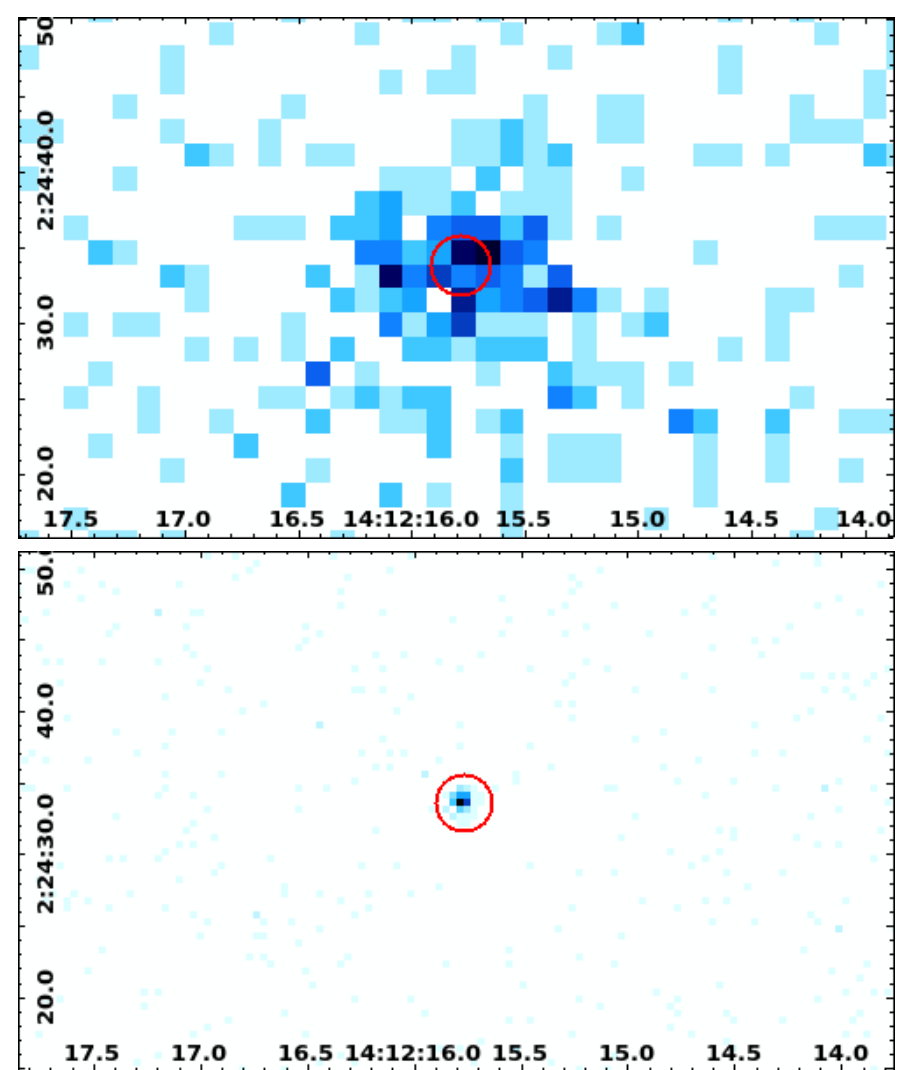

Fig. 1. X-ray images of CU Vir; XMM-Newton pn (top), Chandra ACIS-S (bottom). The red circle ( $2^{\prime \prime}$ radius) denotes the respective optical position.

the absolute positional accuracy $\left(68 \%\right.$ limit is $\left.0.5^{\prime \prime}\right)$. No other $\mathrm{X}$-ray source is present in its vicinity, therefore we attribute the detected X-ray photons to CU Vir in both cases. Whether CU Vir itself is a true single star cannot be determined with X-ray data, but contaminating sources at separations above a few tenths of an arcsecond can be ruled out.

Next, we inspect the X-ray light curves of CU Vir to investigate potential temporal variability of the source. In Fig. 2 we show the background-subtracted light curves over the full energy band of each detector. The low number of detected counts hinders a detailed variability study on short timescales, but strong peaks in X-ray brightness or a pronounced rotational modulation are clearly not present in our data. Overall, a mostly constant or moderately variable source with brightness changes up to a factor of about two are consistent with the data.

CU Vir has a rotation period of about $0.52 \mathrm{~d}(45 \mathrm{ks})$ and each $\mathrm{X}$-ray observation covers slightly more than half a stellar rotation period. To roughly put the X-ray data into the rotational frame of CU Vir, we use the ephemeris given in Trigilio et al. (2011). The radio emission peaks around $\phi=0.35-0.4$ and $\phi=0.75-0.8$, slightly offset but close to the phases of zero longitudinal magnetic field. The stronger and more compact negative southern pole is most visible at $\phi \approx 0.6$, the weaker, positive magnetic pole is most visible at $\phi \approx 0.1$. The XMM-Newton pn exposure starts at $\phi=0.12$ and the radio maximum occurs about $12 \mathrm{ks}$ into the X-ray observation. The X-ray flux during this period is relatively low and, if any, the X-ray brighter phases coincide with the phases when the magnetic pole is most visible. In contrast, the Chandra observation starts around $\phi=0.65$ and covers the rotational phase where the stronger, negative magnetic pole dominates at the beginning of the observation.
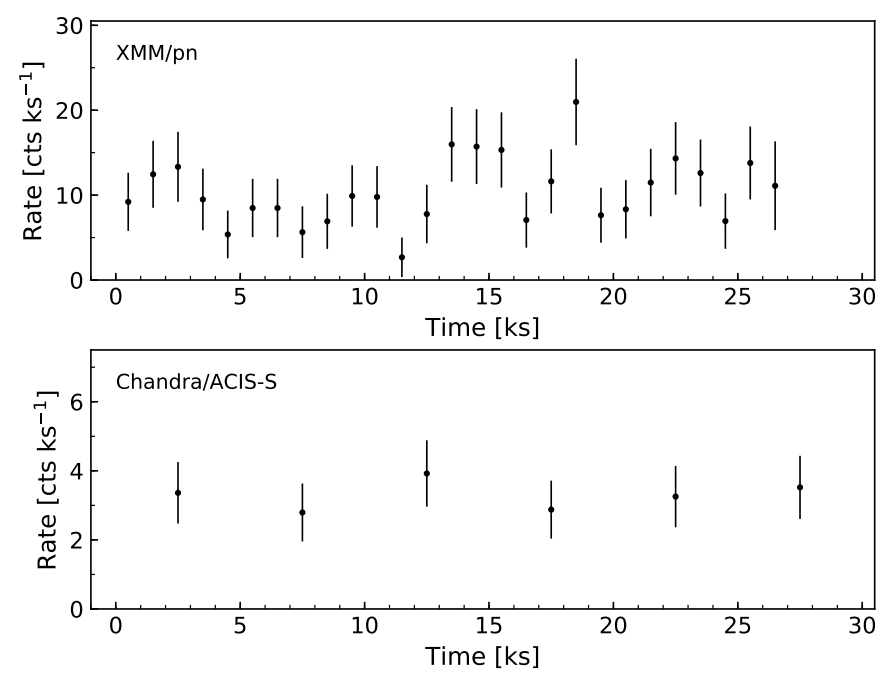

Fig. 2. X-ray light curves of CU Vir; XMM-Newton pn data with $1 \mathrm{ks}$ binning (top) and Chandra ACIS-S with 5 ks binning (bottom).

\section{2. $X$-ray spectra}

Given the absence of detectable strong variations in the X-ray brightness of CU Vir, the spectra were extracted for the full observation each. For the 2011 observation we obtain two XMMNewton spectra, one for the pn detector and one for the two MOS detectors combined; for 2017, the Chandra ACIS-S spectrum is used. The XMM-Newton and Chandra observations are modeled independently, each with a multi-temperature thermal model and a thermal + powerlaw model. Absorption is found to be negligible and is compatible with zero in all applied models; it is therefore neglected in modelling. However due to the interdependence with the emission measure of the cooler plasma the $\mathrm{X}$-ray spectra are not suited to deriving meaningful constraints. Similarly, Kochukhov et al. (2014) find no evidence for circumstellar material in the vicinity of CU Vir in their analysis of hydrogen lines. Abundances were set to solar values as likewise any potential deviation cannot be meaningfully explored with the data. The X-ray spectra and respective best fit models are shown in Fig. 3.

For X-ray spectra with CCD type spectral resolution and moderate signal-to-noise ratios (S/Ns), the two models look quite similar and not surprisingly they achieve a similar quality in fitting the spectra. To describe the XMM-Newton pn + MOS spectra we find a three-temperature or a singletemperature + power-law model sufficient. The Chandra ACIS spectrum can be described with a two-temperature model or a single temperature plus powerlaw model, whereas the thermal component is poorly constrained and was fixed at $1 \mathrm{keV}$, that is, roughly the XMM-Newton value. While several derived spectral model parameters are only moderately constrained, the general spectral trends are robust and independent of the used dataset or modeling approach. A distance of $79 \mathrm{pc}$ is adopted for CU Vir to convert the X-ray fluxes to luminosities, and for the applied models we find $F_{\mathrm{X}}=3.8 \times 10^{-14} \mathrm{erg} \mathrm{cm}^{-2} \mathrm{~s}^{-1}$ as mean value in the $0.2-10.0 \mathrm{keV}$ band. The X-ray luminosities used to study long-term variability are calculated additionally in the energy range $0.5-5.0 \mathrm{keV}$, which is where fluxes are sufficiently well constrained by both datasets. Our modeling results are summarized in Table 2.

Comparing the XMM-Newton observation in 2011 with the one from Chandra in 2017, the spectra are overall similar and the derived model parameters, such as plasma temperatures and/or 

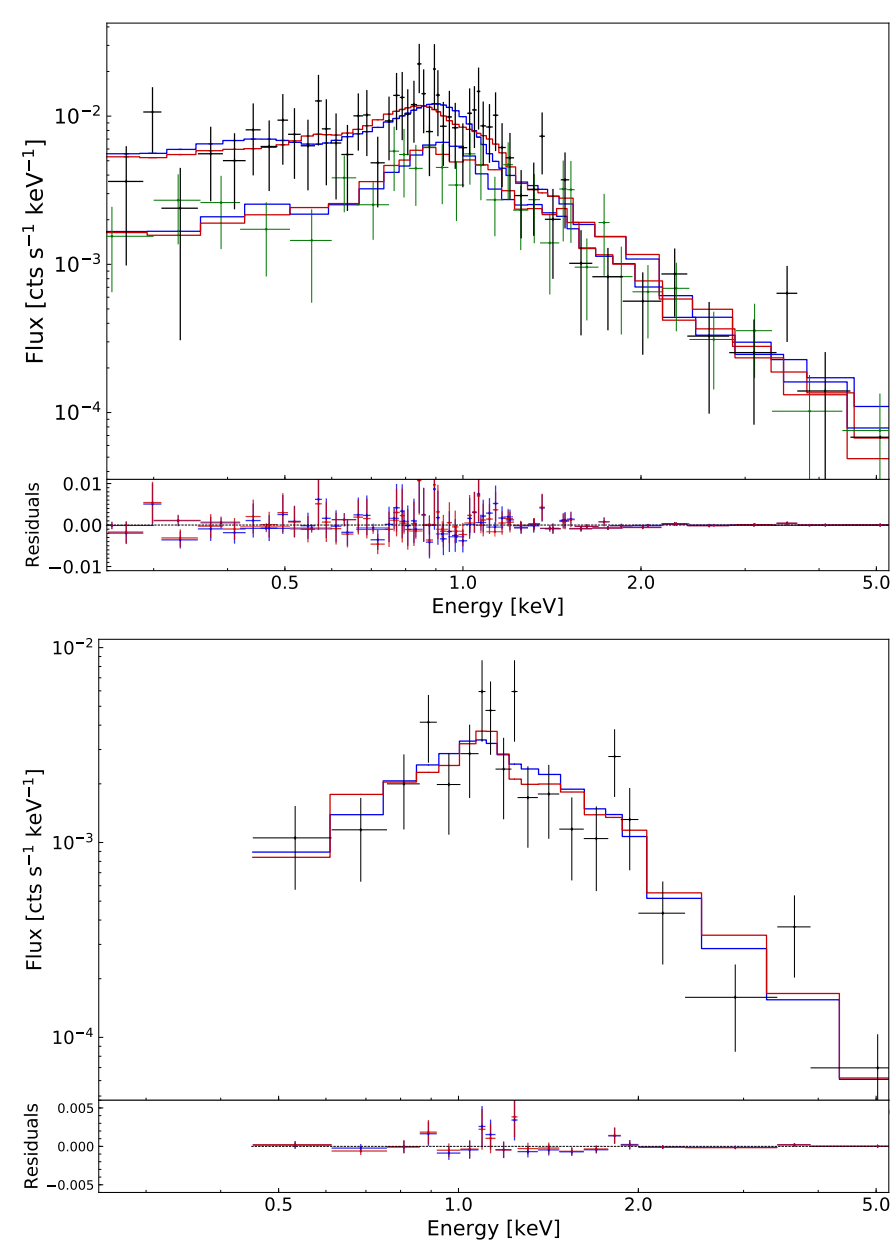

Fig. 3. X-ray spectra of $\mathrm{CU}$ Vir binned to a minimum of five counts per bin; XMM-Newton $\mathrm{pn} / \mathrm{black}+\mathrm{MOS} / \mathrm{green}$ (top) and Chandra ACIS-S (bottom) with thermal (red) and thermal + nonthermal (blue) model respectively. Residuals are shown in the sub-panels.

power-law slope are, except for the normalization, within errors fully consistent between the two observations.

Independent of choice of spectral model (purely thermal or thermal + power-law), the observed source flux is strongly dominated by the hottest plasma or the power-law component. The thermal models are dominated by hot plasma at temperatures of 20-30 MK; cooler plasma at temperatures less than $10 \mathrm{MK}$ is present but found to be a minor contributor at about $30 \%$. The three-temperature modeling of the XMM-Newton data indicates the presence of plasma with a broad temperature distribution below $10 \mathrm{MK}$. While the detailed shape of the emission measure distribution (EMD) is poorly constrained, the full EMD is always dominated by hot plasma. The average plasma temperatures $T_{\mathrm{X}}$ are in the $22-25 \mathrm{MK}$ range in all thermal models.

In thermal + nonthermal models, the emission is dominated by a power-law component with a slope in the range of 1.9-2.2 in both datasets. The modeled plasma temperature of the thermal component is about $10 \mathrm{MK}$ and is thus slightly higher than the average temperature derived for the cooler component(s) in the purely thermal model. Additional cooler plasma components might be present, but are at most a minor contributor. While the thermal component is poorly constrained in the Chandra data, a pure power-law model results for the XMM-Newton data in a very poor fit (C-Stat: 94.1/83) and can be virtually ruled out. As a cross-check we performed a "goodness" Monte-Carlo test in Xspec on the XMM-Newton spectra binned to a minimum of 15 counts. In a run with 1000 simulations $96.80 \%$ of the model realizations have a better test statistics than the data, confirming the low probability of the single power-law model.

Taking the results derived from the XMM-Newton observation, the power-law component contributes about $70 \%$ of the flux in the $0.2-10.0 \mathrm{keV}$ band and $95 \%$ to the flux above $2.0 \mathrm{keV}$. Even more extreme is the Chandra observation, where we find a roughly $30 \%$ higher X-ray luminosity $(0.5-5.0 \mathrm{keV}$ band) that is mostly caused by an increase of the emission measure in the hottest plasma component or the normalization of the powerlaw component that contributes here about $90 \%$. Whether the flux difference between 2011 and 2017 is associated to longterm variability or is due to the fact that the observations have a different phase coverage remains an open question. Overall, the re-detection with Chandra at a comparable brightness of $L_{\mathrm{X}}=2-3 \times 10^{28} \mathrm{erg} \mathrm{s}^{-1}$ establishes CU Vir as a persistent and hard stellar X-ray source.

\subsection{Activity ratios}

Using $L_{\text {bol }} \approx 100 L_{\odot}$ (Kochukhov et al. 2014) we obtain $\log L_{\mathrm{X}} / L_{\mathrm{bol}}=-7.1$. The activity ratio $\log L_{\mathrm{X}} / L_{\mathrm{bol}}$ of CU Vir is two orders higher than the value derived from the correlation obtained by Nazé et al. (2014) for magnetic early/mid B stars, which predicts $\log L_{\mathrm{X}} / L_{\mathrm{bol}} \approx-9.2$ from luminosity scaling. Adopting the estimated mass-loss rate of $\dot{M} \approx 10^{-12} M_{\odot} \mathrm{yr}^{-1}$ from Leto et al. (2006), one predicts again $\log L_{\mathrm{X}} / L_{\mathrm{bol}} \approx-9.2$. Even considering the scatter of about one order of magnitude that is present within these relations, CU Vir appears as a strong $\mathrm{X}$-ray active star if compared to more massive stars powered solely by magnetically channeled wind shocks.

Using the average radio spectral luminosity of $L_{\mathrm{rad}} \approx 3 \times$ $10^{16} \mathrm{erg} \mathrm{s}^{-1} \mathrm{~Hz}^{-1}$ (Leto et al. 2006), we obtain $\log L_{\mathrm{X}} / L_{\mathrm{rad}}=$ 12.0. The derived $X$-ray/radio brightness ratio strongly violates the Guedel-Benz relation, which predicts $\log L_{\mathrm{X}} / L_{\mathrm{R}}=15.5$ for coronal emission (Güdel \& Benz 1993). Even considering the scatter of about half an order of magnitude that is present within these relations, CU Vir appears as a strong radio-active star if compared to coronal sources that are powered by magnetic activity.

\section{Discussion}

The derived X-ray properties of CU Vir and potential X-ray generating mechanisms are discussed in the following.

\subsection{Intrinsic versus extrinsic emission}

The sharp Chandra PSF and good positional match make a chance alignment with an unrelated object very unlikely. This is especially true at its galactic latitude of $+58.6 \mathrm{deg}$ and its X-ray spectra without any hints of absorption of the line of sight that require multiple thermal or thermal + nonthermal components in modeling. In contrast, a very close and so far undiscovered companion to CU Vir is not ruled out by our data. Given the measured X-ray luminosity of $\log L_{X}=28.4$, a late-type star is a viable option. However, the extraordinarily hard X-ray spectrum goes against this hypothesis for our target.

Even active $M$ dwarfs emitting several times $10^{28} \mathrm{erg} \mathrm{s}^{-1}$ in X-rays, that is, those that are comparable or moderately X-ray-brighter than CU Vir, have significantly cooler coronae with average temperatures of 6-8 MK and SEDs that peak around 8 MK (e.g., Robrade \& Schmitt 2005). Stars that possess coronae with average temperatures at quasi-quiescent level as observed for 
Table 2. Spectral fit results for CU Vir@79 pc, XMM-Newton: pn + MOS, Chandra: ACIS-S.

\begin{tabular}{|c|c|c|c|c|}
\hline \multirow[t]{2}{*}{ Parameter } & \multicolumn{3}{|c|}{ Observation } & \multirow[t]{2}{*}{ Unit } \\
\hline & \multicolumn{2}{|c|}{ XMM-2011 } & Chandra-2017 & \\
\hline kT1 & $0.12_{-0.09}^{+0.25}$ & $0.72_{-0.15}^{+0.12}$ & $0.23_{-0.14}^{+0.72}$ & $\mathrm{keV}$ \\
\hline $\mathrm{kT} 2$ & $0.76_{-0.14}^{+0.29}$ & $2.54_{-0.60}^{+1.30}$ & $2.65_{-0.69}^{+0.14}$ & $\mathrm{keV}$ \\
\hline kT3 & $2.62_{-0.69}^{+1.95}$ & -0.00 & $\begin{array}{r}-0.09 \\
-\end{array}$ & $\mathrm{keV}$ \\
\hline EM1 & $0.23_{-0.20}^{+0.49}$ & $0.27_{-0.08}^{+0.09}$ & $0.50_{-0.40}^{+0.37}$ & $10^{51} \mathrm{~cm}^{-3}$ \\
\hline EM2 & $0.28^{+0.090}$ & $1.13^{-0.22}$ & $1.67^{-0.34}$ & $10^{51} \mathrm{~cm}^{-3}$ \\
\hline EM3 & $1.04_{-0.22}^{+0.23}$ & $\begin{array}{r}-0.22 \\
-\end{array}$ & $\begin{array}{r}-0.32 \\
-\end{array}$ & $10^{51} \mathrm{~cm}^{-3}$ \\
\hline C-Stat (d.o.f.) & $45.6(79)$ & $49.7(81)$ & $19.5(16)$ & \\
\hline$L_{\mathrm{X}}(0.2-10.0 / 0.5-5.0 \mathrm{keV})$ & $2.8 / 1.9$ & $2.5 / 2.0$ & $-/ 2.6$ & $10^{28} \mathrm{erg} \mathrm{s}^{-1}$ \\
\hline $\mathrm{kT}$ & \multicolumn{2}{|c|}{$0.92_{-020}^{+0.11}$} & 1.0 & $\mathrm{keV}$ \\
\hline EM & \multicolumn{2}{|c|}{$0.33_{-0.11}^{+0.12}$} & $0.32_{-0.3}^{+0.3}$ & $10^{51} \mathrm{~cm}^{-3}$ \\
\hline$\alpha$ & \multicolumn{2}{|c|}{$2.0_{-02}^{+0.2}$} & $2.4_{-0.5}^{+0.3}$ & [PhoIndex] \\
\hline Norm (at $1 \mathrm{keV}$ ) & \multicolumn{2}{|c|}{$4.5_{-1.0}^{+1.0}$} & $9.7_{-2.8}^{+2.3}$ & $10^{-6} \mathrm{ph} \mathrm{keV}^{-1} \mathrm{~cm}^{-2}$ \\
\hline C-Stat (d.o.f.) & \multicolumn{2}{|c|}{$50.4(81)$} & $18.4(17)$ & \\
\hline$L_{\mathrm{X}}(0.2-10.0 / 0.5-5.0 \mathrm{keV})$ & \multicolumn{2}{|c|}{$2.9 / 1.9$} & $-/ 2.5$ & $10^{28} \mathrm{erg} \mathrm{s}^{-1}$ \\
\hline
\end{tabular}

CU Vir exist, however these have so far only been observed in stars with X-ray luminosities around or exceeding the $\log L_{X}=30$ level, that is, objects that show one hundred times more X-ray luminosity. These luminosities are ruled out, as the distance to CU Vir and thereby flux to luminosity conversion, is well established. The recent Gaia DR2 gives $d=72 \pm 2 \mathrm{pc}$, even reducing the above luminosities by about $20 \%$.

The shape of the observed X-ray light curves, each obtained over several tens of kiloseconds, also clearly argues against an origin in a very strong flare that could produce sufficient amounts of correspondingly hot plasma. Furthermore, the repeated X-ray detection at a similar brightness and spectral hardness in observation separated by several years suggest that the derived X-ray properties reflect the typical state for CU Vir.

Overall, coronal emission from a stellar companion might contribute, but is an unlikely explanation for the bulk of X-rays that are observed from CU Vir. As the possibility of a chance alignment with an extragalactic or galactic counterpart is negligible, intrinsic mechanisms that are capable of generating the observed X-ray emission are considered to be the most likely explanation for the X-ray detection.

\subsection{CU Vir in context of magnetic intermediate-mass stars}

The strong violation of both stellar scaling relations by CU Vir highlights its outstanding character also at X-ray energies, and in combination with its moderate X-ray luminosity and hard X-ray spectrum, it is so far unique in the late-B/earlyA star regime. Although several stars in the MCP sample presented in Robrade (2016) have similar characteristics in at least one or two of the relevant parameters, indicating similarities in their X-ray generating mechanism, none is as extreme as CU Vir.

Even typical magnetic early-B stars with similar $\log L_{\mathrm{X}} / L_{\mathrm{bol}}$ typically have significantly cooler/softer X-ray emission (Oskinova et al. 2011). Nevertheless, comparably hard X-ray spectra combined with high $\log L_{\mathrm{X}} / L_{\text {bol }}$ are also known for a few magnetic early-B stars like HD 182180/HR 7355 and HD 142184/HR 5907 (Nazé et al. 2014; Leto et al. 2017, 2018) or $\sigma$ Ori E (Sanz-Forcada et al. 2004), however these have X-ray
Table 3. CU Vir vs. early Bp stars.

\begin{tabular}{lccc}
\hline \hline & CU Vir & HR 5907 & HR 7355 \\
\hline$M_{*}\left(M_{\odot}\right)$ & 3.1 & 5.5 & 6.0 \\
$P_{\text {rot }}(\mathrm{d})$ & 0.52 & 0.51 & 0.52 \\
$B_{\mathrm{p}}(\mathrm{kG})$ & 3.8 & 15.7 & 11.6 \\
$\log L_{\mathrm{X}}\left(\mathrm{erg} \mathrm{s}^{-1}\right)$ & 28.4 & 30.1 & 30.0 \\
$\mathrm{kT} / \alpha$ & $0.9 / 2.0$ & $1.0 / 1.6$ & $1.0 / 1.7$ \\
$\log L_{\mathrm{X}} / L_{\mathrm{bol}}$ & -7.1 & -6.4 & -6.5 \\
$\log L_{\mathrm{X}} / L_{\mathrm{rad}}$ & 12.0 & 11.8 & 12.0 \\
\hline
\end{tabular}

Notes. CU Vir (this work), HR 5907 (Leto et al. 2018), HR 7355 (Leto et al. 2017).

luminosities that are above that of CU Vir by factors of several tens or even hundreds.

Intriguingly, the X-ray spectral properties are also quite similar between CU Vir and the fast rotating Bp stars HR 7355 and HR 5907 (Leto et al. 2017, 2018). Although the X-ray luminosity of these Bp stars is one to two orders of magnitude higher, the basic X-ray spectral properties and X-ray activity as well as $\mathrm{X}$-ray/radio ratios are comparable. A discussion relating to the auroral model proposed for these stars is given in the following section. In Table 3 we compare relevant parameters to highlight their similarities and differences.

\subsection{Purely thermal versus thermal plus nonthermal X-rays}

In $\mathrm{Ap} / \mathrm{Bp}$ stars the stellar magnetosphere offers several mechanisms capable of producing X-ray emission. The strongly magnetic star CU Vir possesses a primarily dipolar-like nonaxisymmetric magnetic structure. Thermal X-ray plasma will be naturally created in wind shocks via the MCWS mechanism, but the weaker winds in late-B/early-A stars with terminal velocities of $V_{\text {inf }} \approx 600 \mathrm{~km} \mathrm{~s}^{-1}$ are expected to create plasma with post-shock temperatures of a few up to about $10 \mathrm{MK}$ (e.g., ud-Doula et al. 2014). The "classical" MCWS plasma is therefore a suitable candidate for the cooler plasma component(s) in our spectral models, but insufficient to explain the hot, dominant component around $30 \mathrm{MK}$. 
Overall, the main characteristics of CU Vir derived here, that is, faint but hard $\mathrm{X}$-ray emission, are virtually the exact opposite of the original motivation for the development of the MCWS model by Babel \& Montmerle (1997) to explain the ROSAT data of the A0p star IQ Aur; i.e., bright but soft X-ray emission.

Clearly, a mechanism that, depending on the adopted model, is producing the hottest plasma or nonthermal component is needed. Furthermore, this component is not a small extra, but actually the dominant contribution to the observed X-ray emission; comparing results from 2011 (XMM-Newton) with those from 2017 (Chandra) it is likely also the most variable component.

The very hot plasma could arise from activity-like phenomena associated with magnetic spots or plasma captured in the magnetosphere, where, in addition, re-connection events might occur in the disk-like structure, for example during break-out or infall events. However, compared to similar Ap/Bp stars like the well studied IQ Aur (Robrade \& Schmitt 2011), the required plasma temperatures of CU Vir are extreme. We find quasi-quiescent $\mathrm{X}$-rays for IQ Aur with $\log L_{\mathrm{X}} \approx 29.6 \mathrm{erg} \mathrm{s}^{-1}$ and $T_{\mathrm{X}} \approx 8 \mathrm{MK}$ and for CU Vir with $\log L_{\mathrm{X}} \approx 28.4 \mathrm{erg} \mathrm{s}^{-1}$ and $T_{\mathrm{X}} \approx 25 \mathrm{MK}$. Similarly hard X-ray spectra are only seen during a flare event in IQ Aur, which is associated with very hot thermal plasma at several tens of megakelvin as deduced from the detection of a strong $6.7 \mathrm{keV}$ Fe xxv emission line complex. However, in IQ Aur this is a transient phenomenon, whereas the very hot component is clearly associated with the quasi-quiescent state of CU Vir.

An alternative scenario, involving a nonthermal component that describes auroral X-ray emission, was proposed by Leto et al. (2017), put forward to explain the X-ray emission from fast-rotating magnetic early B star HR 7355. It suggests, that besides the thermal emission originating from the MCWS that heats plasma up to a few megakelvin, nonthermal emission should be present in fast-rotating magnetic Ap/Bp stars. In this scenario the X-ray emission originates from the nonthermal electrons upon impact on the stellar surface. The electron population would be identical to the one that is also responsible for the gyrosynchrotron radio emission. The nonthermal electron population able to produce the incoherent gyrosynchrotron emission of CU Vir has a power-law energy distribution (Leto et al. 2006) with a low-energy cutoff of $\approx 100 \mathrm{keV}$ (Trigilio et al. 2004). Such nonthermal electrons, accelerated by magnetic reconnection events occurring in the current-sheet regions located far from the star at about 15 stellar radii, precipitate toward the stellar surface. The energy budget of these precipitating electrons is compatible with what is observed during the Sun flares, where also hard X-ray emission is detected at the footprints of the magnetic loops (see Aschwanden 2002, and references therein). In this case the hard X-ray component would be a power-law component generated by thick target bremsstrahlung emission from the nonthermal electrons. Indeed, CU Vir could be understood as a down-scaled version of HR 7355, whose radio luminosity as well as its X-ray luminosity is about a factor 30 higher. What causes these phenomena in CU Vir and similar objects remains unknown, but fast rotation and strong dipolar fields have been identified as common attributes. Further hard X-ray observations at photon energies higher than $5 \mathrm{keV}$ could be very useful to definitively confirm this scenario.

The X-ray spectrum of CU Vir alone is not able to definitely support the scenario where the hard X-ray component has auroral origin. The thermal and the nonthermal scenario are both viable and even both may be at work simultaneously. Furthermore, the thermal component in itself may be a composite that is partly MCWS, magnetospheric activity, or reprocessed auroral emission in nature. However, in any case some extraordinary
X-ray generation mechanism must be present in any intrinsic emission scenario.

\section{Summary}

We detect persistent $\mathrm{X}$-ray emission from the A0p stellar radio pulsar CU Vir. Its main characteristics can be summarized as follows.

1. The X-ray luminosity is moderate with $L_{\mathrm{X}} \approx 3 \times 10^{28} \mathrm{erg} \mathrm{s}^{-1}$; light curves show only minor variability.

2. The X-ray spectra are very hard; their modeling requires multi-thermal models dominated by hot plasma at $25 \mathrm{MK}$ or thermal + nonthermal plasma components.

3. The X-ray emission is very likely intrinsic; modified MCWS models, for example, including aurorae, are promising, but details of its generating mechanism remain unknown.

4. The XMM-Newton (2011) and Chandra (2017) data give similar X-ray properties, indicating that these are quite stable and persistent.

5. The A0p type star CU Vir is in essence a down-scaled version of the early-B type MCP stars characterized by a similar $\mathrm{X}$-ray spectrum and X-ray/radio luminosity ratio.

The X-ray and radio features observed in CU Vir can be explained in general terms by the scenario proposed in Leto et al. (2017); it therefore holds in both the early-B and late-B/early-A fast rotating MCP stars. As a future outlook we plan to perform combined X-ray and radio data modeling and to extend the simulations of the radio emission of CU Vir up to millimeter wavelengths.

Acknowledgements. This work is based on observations obtained with Chandra and XMM-Newton. J. R. and LMO acknowledge support from the DLR under grant 50QR1605 (JR) and FKZ50OR1508 (LMO). LMO acknowledges partial support by the Russian Government Program of Competitive Growth of Kazan Federal University.

\section{References}

Arnaud, K. A. 1996, ASP Conf. Ser., 101, 17

Aschwanden, M. J. 2002, Space Sci. Rev., 101, 1

Babcock, H. W. 1958, ApJS, 3, 141

Babel, J., \& Montmerle, T. 1997, A\&A, 323, 121

de la Calle, I. 2018, Users Guide to the XMM-Newton Science Analysis System (ESA: XMM-Newton SOC)

Fruscione, A., McDowell, J. C., \& Allen, G. E. 2006, Proc. SPIE, 6270, 62701V Güdel, M., \& Benz, A. O. 1993, ApJ, 405, L63

Kochukhov, O., et al. (MiMeS Collaboration) 2014, A\&A, 565, A83

Krtička, J., Mikulášek, Z., Lüftinger, T., et al. 2012, A\&A, 537, A14

Krtička, J., Mikulášek, Z., Henry, G. W., Kurfürst, P., \& Karlický, M. 2017, MNRAS, 464, 933

Leto, P., Trigilio, C., Buemi, C. S., Umana, G., \& Leone, F. 2006, A\&A, 458, 831

Leto, P., Trigilio, C., Oskinova, L., et al. 2017, MNRAS, 467, 2820

Leto, P., Trigilio, C., Oskinova, L. M., et al. 2018, MNRAS, 476, 562

Mikulášek, Z., Krtička, J., Henry, G. W., et al. 2011, A\&A, 534, L5

Nazé, Y., Petit, V., Rinbrand, M., et al. 2014, ApJS, 215, 10

Oskinova, L. M., Todt, H., Ignace, R., et al. 2011, MNRAS, 416, 1456

Robrade, J. 2016, Adv. Space Res., 58, 727

Robrade, J., \& Schmitt, J. H. M. M. 2005, A\&A, 435, 1073

Robrade, J., \& Schmitt, J. H. M. M. 2011, A\&A, 531, A58

Sanz-Forcada, J., Franciosini, E., \& Pallavicini, R. 2004, A\&A, 421, 715

Smith, R. K., Brickhouse, N. S., Liedahl, D. A., \& Raymond, J. C. 2001, ApJ, 556, L91

Trigilio, C., Leto, P., Leone, F., Umana, G., \& Buemi, C. 2000, A\&A, 362, 281

Trigilio, C., Leto, P., Umana, G., Leone, F., \& Buemi, C. S. 2004, A\&A, 418, 593

Trigilio, C., Leto, P., Umana, G., Buemi, C. S., \& Leone, F. 2011, ApJ, 739, L10

ud-Doula, A., Owocki, S., Townsend, R., Petit, V., \& Cohen, D. 2014, MNRAS, 441, 3600

van Leeuwen, F. 2007, A\&A, 474, 653 